\title{
Possible correlations between the psychological state and excessive innate immunity responses in ulcerative colitis
}

\author{
Ilias Vlachos ${ }^{1 *}$, Maria Coccossis ${ }^{2}$, Maria Tsopanomichalou ${ }^{3}$, Christos Christoidoulou $^{4}$, George Papadimitriou$^{2}$, \\ Efstathios Patsouris ${ }^{1}$, Calypso Barbatis ${ }^{3}$, Polyxeni Nicolopoulou-Stamati ${ }^{1}$ \\ From $1^{\text {st }}$ International Congress on Neurobiology and Clinical Psychopharmacology \\ and European Psychiatric Association Conference on Treatment Guidance \\ Thessaloniki, Greece. 19-22 November 2009
}

\section{Background}

Inflammatory bowel diseases (IBD), including ulcerative colitis (UC) and Crohn's disease (CD), belong to the autoimmune disorders in the sense that an excessive response of the immune system(both innate and acquired) towards commensal microbial flora of the intestinal mucosa is involved in their pathogenesis. The progress of IBD is unknown, characterized by periods of exacerbation and quiescence. Depression and anxiety seem to coincide with relapse of IBD and further research is needed for the clarification of this correlation.

\section{Aim}

To further investigate the relationship between the psychological state of UCpatients and the gravity of their biopsy during relapse.

\section{Materials and methods}

29 UC patients, hospitalized in two general hospitals for the investigation of a possible relapse of their disease were examined. Methods: Four self- report inventories (Hospital Anxiety and Depression Scale-HADS, Zung Depression Scale, State Trait Anxiety Inventory Form1 and Form 2/STAI1/STAI2) were administered to the patients and the scores were correlated with the severity of parameters of their corresponding biopsies.

\section{Results}

Positive correlations were observed between the degree of anxiety and depression in the questionnaires and the activation of innate immunity (polymorphonuclear leucocytes and macrophages) in the biopsies of UC patients

\section{Conclusions}

Our findings suggest correlations between the psychological state of UC patients and the intensity of their innate immune response perpetuating inflammation.

\section{Author details}

'Pathology Department, Medical School Athens University, Athens, Greece. ${ }^{2}$ First Psychiatry Department, Medical School Athens University, Athens, Greece. ${ }^{3}$ Pathology Department, Hellenic Red Cross Hospital, Athens, Greece. ${ }^{4}$ Second Psychiatry Department, Medical School Athens University, Athens, Greece.

\section{Published: 22 April 2010}

References

1. Shih DQ, Targan SR, McGovern D: Recent advances in IBD pathogenesis: genetics and immunobiology. Curr Gastroenterol Rep 2008, 10(6):568-75.

2. Mikocka-Walus AA, Turnbull DA, Moulding NT, Wilson IG, Andrews JM,

Holtmann GJ: Controversies surrounding the comorbidity of depression and anxiety in inflammatory bowel disease patients: a literature review. Inflamm Bowel Dis 2007, 13(2):225-34.

3. Hisamatsu T, Ogata H, Hibi T: Innate immunity in inflammatory bowel disease: state of the art. Curr Opin Gastroenterol 2008, 24(4):448-454.

doi:10.1186/1744-859X-9-S1-S182

Cite this article as: Vlachos et al.: Possible correlations between the psychological state and excessive innate immunity responses in ulcerative colitis. Annals of General Psychiatry 2010 9(Suppl 1):S182.

'Pathology Department, Medical School Athens University, Athens, Greece 